ISSN 0103-9954

\title{
ESTRUTURA DO COMPONENTE DE REGENERAÇ̃̃O NATURAL E ARBÓREO DE DOIS MANGUEZAIS NO ESTADO DO PARANÁ
}

\author{
STRUCTURE OF ADULT TREES AND REGENERATION COMPONENT OF TWO MANGROVE \\ FORESTS OF PARANÁ
}

\author{
Ana Paula Lang Martins Madi ${ }^{1}$ Maria Regina Torres Boeger ${ }^{2}$ Letícia Larcher ${ }^{3}$ \\ Andressa Pelozo $^{4}$ Carolina Sereneski ${ }^{5}$ Carlos Bruno Reissmann ${ }^{6} \quad$ André Andrian Padial $^{7}$
}

\section{RESUMO}

Esse estudo avaliou comparativamente os parâmetros fitossociológicos da regeneração natural e da vegetação arbórea em dois manguezais distintos no Estado do Paraná, um pertencente à Baía de Paranaguá (manguezal de Antonina) e outro à Baía de Guaratuba (manguezal de Guaratuba). Em cada área, três subáreas foram marcadas e amostradas cinco parcelas de 10 x $10 \mathrm{~m}$ para avaliação do componente arbóreo e quinze subparcelas de 1 x $1 \mathrm{~m}$ para avaliação da regeneração natural. Avicennia schaueriana, Rhizophora mangle e Laguncularia racemosa exibiram domínio e densidade relativa distintos entre as áreas. Manguezais de Antonina e Guaratuba diferiram tanto na estrutura da regeneração natural como no componente arbóreo. A Análise de Coordenadas Principais ( $\mathrm{PCoA})$ mostrou que as diferenças entre as áreas em relação às variáveis abióticas do solo estão fortemente relacionadas com os parâmetros estruturais do componente arbóreo dos manguezais. Os fatores edáficos dos manguezais têm grande importância na separação entre as áreas, já que reflete na capacidade de adaptação das plantas.

Palavras-chave: fitossociologia; mangue; salinidade; solo.

\begin{abstract}
We analyzed the structures of two mangrove forests of Paraná (Brazil) (Antonina Bay and Guaratuba Bay), considering adult trees and regeneration component. In each area, we marked three sub-areas and sampled five plots of $10 \times 10 \mathrm{~m}$, equivalent to 0.05 ha to adults trees and fifteen $1 \times 1 \mathrm{~m}$ sub-plots to assess the regeneration component. Avicennia schaueriana, Rhizophora mangle and Laguncularia racemosa exhibited distinct dominance and relative density between the areas. Mangroves of Antonina and Guaratuba differed both in the structure of natural regeneration as the tree component. The Principal Coordinates Analysis $(\mathrm{PCoA})$ show that differences between areas in relation to abiotic variables of soil are strongly related to the structural parameters of trees. The different types of mangrove soil are the most significant factor in the differentiation between the mangrove areas since influences the salinity values and consequent adaptability of plants.
\end{abstract}

Keywords: forest structure; mangrove; salinity; soil.

1 Química Ambiental, Dr $^{\mathrm{a}}$., Centro Politécnico, Universidade Federal do Paraná, Caixa Postal 19073, CEP 81531-980, Curitiba (PR), Brasil. langmartins@hotmail.com

2 Bióloga, Dra ., Professora do Departamento de Botânica, Centro Politécnico, Universidade Federal do Paraná, Caixa Postal 19073, CEP 81.531-980, Curitiba (PR), Brasil. rboeger@ufpr.br

3 Bióloga, Doutoranda em Ecologia e Conservação, Centro Politécnico, Universidade Federal do Paraná, Caixa Postal 19073, CEP 81531-980, Curitiba (PR), Brasil. leticialarcher@gmail.com

4 Bióloga, MSc., Doutoranda em Biologia Comparada, Universidade Estadual de Maringá, Av. Colombo, 5790 PGB - Bloco G-80, Sala 201, CEP 87020-900, Maringá (PR), Brasil. pelozo.andressab@gmail.com

5 Bióloga, MSc., Centro Politécnico, Universidade Federal do Paraná, Caixa Postal 19073, CEP 81.531-980, Curitiba (PR), Brasil. carolsereneski@hotmail.com

6 Engenheiro Florestal, Dr., Professor do Departamento de Solos, Universidade Federal do Paraná, Rua dos Funcionários, 1540, CEP 80035-020 , Curitiba (PR), Brasil. reissman@ufpr.br

7 Biólogo, Dr., Professor do Departamento de Botânica, Centro Politécnico, Universidade Federal do Paraná, Caixa Postal 19031, CEP 81531-980, Curitiba (PR), Brasil. aapadial@gmail.com 


\section{INTRODUÇÃO}

Os manguezais são ecossistemas costeiros de transição entre ambientes terrestre e marinho, característico de regiões tropicais e subtropicais, sujeito ao regime das marés (SCHAFFERNOVELLI, 1995). No Brasil, sua extensão abrange desde o Oiapoque $\left(04^{\circ} 30^{\prime} \mathrm{N}\right)$ no Amapá, até a região Sul, em Laguna (283's), SC (SCHAEFFERNOVELLI, 2000). São considerados ambientes produtivos e possuem capacidade de exportação de detritos orgânicos para os sistemas costeiros adjacentes (SCHAEFFER-NOVELLI, 1991).

Os manguezais são classificados como uma Formação Pioneira de Influência Fluviomarinho, correspondendo ao "complexo edáfico de primeira ocupação" (IBGE, 1992). Em função da complexidade de interações abióticas, os manguezais do litoral brasileiro foram classificados por Schaeffer-Novelli et al. (1990) em oito unidades fisiográficas. O litoral do Paraná está incluído na Unidade VII (Cabo Frio a Torres), no qual os manguezais são formações florestais dominantes nas ilhas planas das baías e embocaduras dos rios (BIGARELLA, 1946; MAACK, 1981).

Nos manguezais brasileiros ocorrem geralmente quatro espécies: Rhizophora mangle L., Avicennia schaueriana Stapf \& Leechm. ex Moldenke, Avicennia germinans (L.) Stearn e Laguncularia racemosa (L.) C. F. Gaertn e outros gêneros associados como Conocarpus e Spartina (SCHAEFFER-NOVELLI et al., 1990). Estas espécies compartilham características fisiológicas e adaptações especiais que permitem sua ocorrência em ambientes em constante inundação, com alta salinidade e em substratos não consolidados com baixa oxigenação (KATHIRESAN e BINGHAM, 2001).

Laguncularia racemosa é a espécie
dominante nos manguezais paranaenses
(BIGARELLA, 1946), enquanto que Rhizophora mangle ocorre em manguezais em frente ao mar aberto e Laguncularia racemosa em águas menos profundas (MAACK, 1981). Estudos em manguezais da Ilha das Laranjeiras (Guaraqueçaba - PR) mostraram que não há um padrão definido de distribuição das espécies, sugerindo uma heterogeneidade espacial nos mangues paranaenses (SESSEGOLO, 1997),

Tal heterogeneidade parece ser uma resposta à interação de vários fatores abióticos (fatores edáficos e salinidade) e antrópicos que atuam em diferentes escalas espaciais e temporais (BERNINI e REZENDE, 2010). Tanto o componente arbóreo quanto a regeneração natural, representado pelas plântulas e indivíduos jovens, podem apresentar respostas aos diferentes fatores abióticos. Estudos sobre o crescimento das plântulas e a ocorrência dos adultos sugerem que o estabelecimento dos propágulos está relacionado com a tolerância fisiológica das espécies, o tamanho dos propágulos, flutuabilidade e propriedades de dispersão (RABINOWITZ, 1978). Esses fatores interagem para definir a ocorrência dos indivíduos no componente arbóreo, refletindo na distribuição das espécies (KATHIRESAN e BINGHAM, 2001).

Apesar dos manguezais serem considerados Áreas de Proteção Permanente pela Lei Federal 4771/65, ainda são bastante intensas as atividades antrópicas. Assim, os estudos sobre a estrutura dos manguezais representam uma ferramenta importante para o entendimento da relação das espécies de mangue com ambiente local, bem como auxiliar nas ações para à conservação deste ecossistema (SOARES, 1999). Os manguezais no Estado do Paraná ainda não apresentam um alto grau de degradação como ocorre em outros estados brasileiros, mas já existem pressões causadas pela expansão urbana, devido ao polo portuário de Paranaguá e pela indústria de lazer (LANA, 1998).

Nesse contexto, esse estudo teve como objetivo avaliar comparativamente os parâmetros fitossociológicos da regeneração natural e da vegetação arbórea em dois manguezais, uma pertencente à Baía de Paranaguá (manguezal de Antonina) e outra à Baía de Guaratuba (manguezal de Guaratuba), localizados no estado do Paraná. Para isso, investigou-se em quais características abióticas tais áreas diferem e qual a relação dos parâmetros fitossociológicos com as condições edáficas.

\section{MATERIAL E MÉTODOS}

O estudo foi desenvolvido em duas Baías do Estado do Paraná: Paranaguá e Guaratuba. O município de Antonina localiza-se no extremo oeste da Baía de Paranaguá, com uma área de 460 $\mathrm{km}^{2}$. Essa baía sofre forte interferência da ação portuária (Paranaguá e Antonina) e atividades de pesca esportiva. As subáreas foram demarcadas na região média do estuário do Rio Nhundiaquara (Figura 1). A Baía de Guaratuba, com $48,72 \mathrm{~km}^{2}$ de extensão compreende áreas de proteção ambiental. 
As subáreas desta baía foram demarcadas na região média do estuário do Rio dos Pinheiros (Figuras 1A e 1B). Nesse rio, existem atividades de cultivo de ostra e navegação de pequenos barcos.

Os dados climáticos foram cedidos pelo Sistema Meteorológico do Paraná (SIMEPAR) e referem-se ao ano de 2010 (Tabela 1). Os dados edáficos das áreas estudadas foram obtidos a partir do trabalho de Boeger et al. (2011). Em cada área foram marcadas três subáreas para a realização do levantamento fitossociológico e de regeneração natural (A1, A2 e A3, para Antonina e G1, G2 e G3 para Guaratuba). Em cada subárea foram amostradas cinco parcelas de $10 \times 10 \mathrm{~m}$, equivalentes a $0,05 \mathrm{ha}$, de acordo com metodologia proposta por Cintron e Schaeffer-Novelli (1983). A localização das parcelas procurou abranger toda a estreita faixa de mangue, situada nos primeiros $20 \mathrm{~m}$ da borda do manguezal, paralelamente ao corpo hídrico.

Para o estudo da regeneração natural, em cada parcela demarcada para o levantamento fitossociológico do componente arbóreo, foram
$1 \mathrm{~A}$

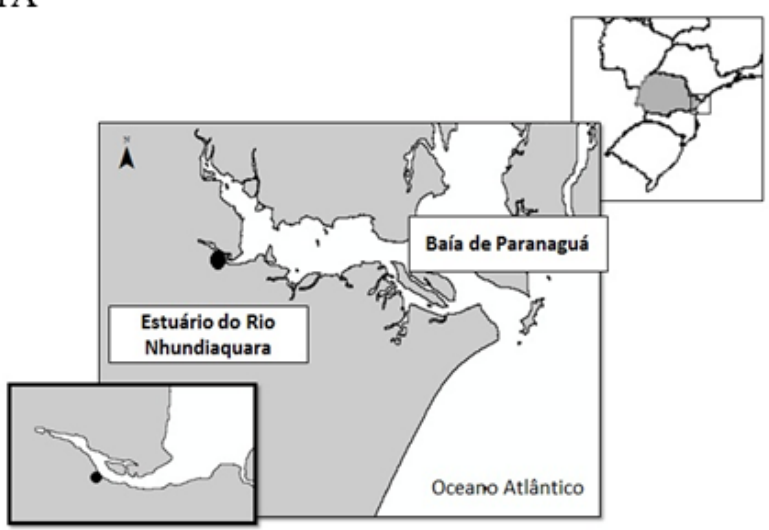

1B

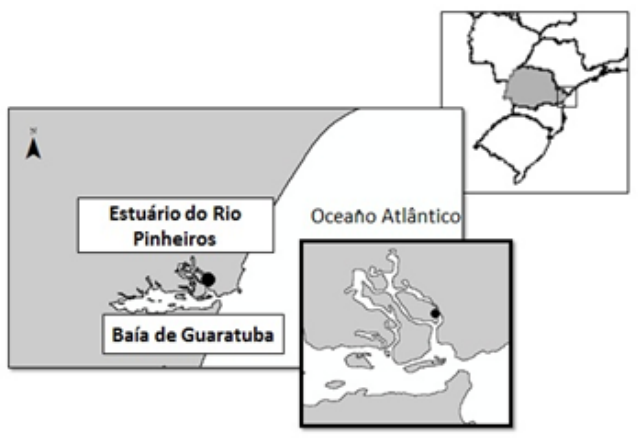

FIGURA 1: 1A - Área de estudo no manguezal do estuário do Rio Nhundiaquara (Antonina - PR); 1B: área de estudo do manguezal do estuário do Rio dos Pinheiros (Guaratuba, PR).

FIGURE 1: 1A - Study area in the mangrove of the estuary of Nhundiaquara River (Antonina, PR); 1B: study area in the mangrove of the estuary of Pinheiros River (Guaratuba, PR).

TABELA 1: Descrição das variáveis abióticas das áreas de estudo: Antonina e Guaratuba, Paraná, Brasil, para o ano de 2010.

TABLE 1: Description of abiotic variables of the studied areas: Antonina and Guaratuba, Paraná, Brasil, for the period of 2010 .

\begin{tabular}{|c|c|c|c|c|c|c|}
\hline & \multicolumn{3}{|c|}{ Antonina } & \multicolumn{3}{|c|}{ Guaratuba } \\
\hline & A1 & A2 & A3 & G1 & G2 & G3 \\
\hline Posicão Geográfica² & $25^{\circ} 29^{\prime} \mathrm{S}$ & $25^{\circ} 29^{\prime} \mathrm{S}$ & $25^{\circ} 29^{\prime} \mathrm{S}$ & $25^{\circ} 50^{\prime} \mathrm{S}$ & $25^{\circ} 49^{\prime} \mathrm{S}$ & $25^{\circ} 49^{\prime} \mathrm{S}$ \\
\hline & $48^{\circ} 42^{\prime} \mathrm{W}$ & $48^{\circ} 42^{\prime} \mathrm{W}$ & $48^{\circ} 41^{\prime} \mathrm{W}$ & $48^{\circ} 34^{\prime} \mathrm{W}$ & $48^{\circ} 34^{\prime} \mathrm{W}$ & $48^{\circ} 34^{\prime} \mathrm{W}$ \\
\hline Temp. $\left({ }^{\circ} \mathrm{C}\right)^{1}$ & 20,5 & 20,5 & 20,5 & 20,8 & 20,8 & 20,8 \\
\hline Clima $^{1}$ & Cfa & $\mathrm{Cfa}$ & Cfa & $\mathrm{Cfa}$ & $\mathrm{Cfa}$ & $\mathrm{Cfa}$ \\
\hline Precipitação anual & 2733 & 2733 & 2733 & 3183 & 3183 & 3183 \\
\hline Tipo de solo 2 & $\begin{array}{c}\text { Organossolo } \\
\text { tiomórfico } \\
\text { sálico } \\
\text { sódico }\end{array}$ & $\begin{array}{l}\text { Organossolo } \\
\text { tiomórfico } \\
\text { sálico } \\
\text { sódico }\end{array}$ & $\begin{array}{c}\text { Organossolo } \\
\text { tiomórfico } \\
\text { sálico } \\
\text { sódico }\end{array}$ & $\begin{array}{l}\text { Gleissolo } \\
\text { tiomórfico } \\
\text { sálico } \\
\text { sódico }\end{array}$ & $\begin{array}{c}\text { Organossolo } \\
\text { tiomórfico } \\
\text { sálico } \\
\text { sódico }\end{array}$ & $\begin{array}{c}\text { Gleissolo } \\
\text { tiomórfico } \\
\text { sálico } \\
\text { sódico }\end{array}$ \\
\hline SAI $(\%)^{2}$ & $\begin{array}{l}\text { Sodico } \\
18,6\end{array}$ & $\begin{array}{l}\text { sodico } \\
13,2\end{array}$ & $\begin{array}{l}\text { Sodico } \\
19\end{array}$ & $\begin{array}{l}\text { Sodico } \\
27,6\end{array}$ & $\begin{array}{l}\text { Sodico } \\
22,2\end{array}$ & $\begin{array}{l}\text { sodico } \\
23,4\end{array}$ \\
\hline $\mathrm{PR}(\mathrm{mV})^{2}$ & -339 & $-347,8$ & $-294,8$ & $-318,6$ & $-308,2$ & $-323,8$ \\
\hline
\end{tabular}

Em que: 'Dados cedidos pelo SIMEPAR ${ }^{2}$ Boeger et al. (2011). Legenda: SAI: salinidade da água intersticial; PR: potencial redox. 
estabelecidas quinze subparcelas com um $\mathrm{m}^{2}$. As subparcelas foram demarcadas sistematicamente, mantendo-se uma distância de um metro entre elas. Em cada subparcela, todos os indivíduos até um metro de altura foram identificados e denominados de plântulas. Para cada indivíduo, o comprimento total e o diâmetro da base do caule foram mensurados com o auxílio de uma fita métrica e um paquímetro, respectivamente. Foram calculados: densidade absoluta (DA), densidade relativa (DR), frequência absoluta (FA) e frequência relativa (FR), de acordo com Mueller-Dombois e Ellenberg (1974).

Para o componente arbóreo, em cada parcela foram medidos o diâmetro a altura do peito (DAP) e a altura de todos os indivíduos vivos e mortos em pé com altura superior a um metro. A altura foi estimada com auxílio de vara graduada (m). O material botânico fértil coletado foi depositado no herbário UPCB (UFPR). A densidade absoluta (DA), densidade relativa (DR), frequência absoluta (FA), frequência relativa (FR), dominância absoluta (DoA), dominância relativa (DoR), valor de importância (VI) e valor de cobertura (VC) foram calculados de acordo com Mueller-Dombois e Ellenberg (1974). Os valores de área basal e densidade foram transformados para hectare.

Para visualizar as diferenças entre as áreas em relação às variáveis abióticas do solo e aos parâmetros fitossociológicos da vegetação arbórea e da estrutura de regeneração natural entre os manguezais foram realizadas Análises de Coordenadas Principais (PCoA) (GOWER, 1966). Como o número de variáveis mensuráveis ultrapassou o número de unidades amostrais, optouse pela PCoA com distância euclidiana. A correlação de Pearson entre as variáveis originais e os escores foi realizada para verificar quais variáveis eram as principais responsáveis pelas diferenças entre os manguezais estudados. As análises foram realizadas usando o Programa "R" (R DEVELOPMENT CORE TEAM, 2009) com os pacotes vegan (OKSANEN et al., 2008) e labdsv (ROBERTS, 2007). Para a construção dos gráficos, foi usado o software STATISTICA (STATSOFT, 2005).

\section{RESULTADOS}

Tanto para o componente arbóreo como para a regeneração natural foram identificadas as seguintes espécies: Avicennia schaueriana Stapf \& Leechmex Moldenke, Rhizophora mangle L. e Laguncularia racemosa (L.) C. F. Gaertn.
Em Antonina, foi amostrado um total de 966 plântulas, sendo 108 de Rhizophora mangle, 857 de Laguncularia racemosa e apenas uma de Avicennia schaueriana. Em Guaratuba, foram identificadas 521 plântulas, sendo 137 de Rhizophora mangle, 200 de Laguncularia racemosa e 184 de Avicennia schaueriana. Laguncularia racemosa apresentou as maiores densidades de plântulas nas subáreas A1, A2, A3 e G3 (Tabela 2), Avicennia schaueriana em G1, mas não foi encontrada nas subáreas A1 e A2, enquanto que Rhizophora mangle teve a maior densidade em G2. As plântulas de Avicennia schaueriana foram mais frequentes em G1, Laguncularia racemosa em A2 e A3 e Rhizophora mangle em A1 e G2 e G3 (Tabela 2).

$\mathrm{O}$ número de indivíduos adultos variou entre as áreas, totalizando 532 na área de Antonina e 475 em Guaratuba. Uma variação acentuada do número de indivíduos entre as subáreas amostradas dentro de cada área também foi observada. Por exemplo, houve uma grande variação entre indivíduos de Avicennia schaueriana para a área de Guaratuba (130 indivíduos em G1 e 16 indivíduos em G3). Em Antonina, a densidade de Rhizophora mangle na subárea $\mathrm{A} 1$ é 2,5 vezes maior em relação à subárea A3 (Tabela 3). Avicennia schaueriana apresentou a maior densidade em G1, enquanto que Rhizophora mangle em A1, A2 e G2 e Laguncularia racemosa em A3 e G3 (Tabela 3). Entre as áreas, também não ocorreu um padrão homogêneo: em Antonina, a espécie com maior densidade foi Laguncularia racemosa, seguida por Rhizophora mangle e Avicennia schaueriana. Em Guaratuba, a espécie com maior densidade foi Avicennia schaueriana, seguida de Rhizophora mangle e Laguncularia racemosa (Tabela 3 ).

Plântulas de Rhizophora mangle apresentaram maior diâmetro e altura em todas as subáreas, com exceção de G3, enquanto que Laguncularia racemosa apresentou os menores valores médios, em ambas as áreas do estudo (Tabela 2). A maioria das plântulas de Rhizophora mangle apresentou altura entre 61 e $80 \mathrm{~cm}$ e Laguncularia racemosa até $20 \mathrm{~cm}$ de altura. Em relação às classes de altura, em todas as subáreas, o maior número de indivíduos ocorreu na classe de $0-20 \mathrm{~cm}$. As exceções foram para A3 e G1 (classe 21 a $40 \mathrm{~cm}$ ) e em G2 (classe $41 \mathrm{a} 60 \mathrm{~cm}$ ), em que houve um número superior a 50 indivíduos.

Os parâmetros estruturais analisados também apresentaram grande variação entre as duas áreas (Tabela 4). A análise estrutural mostrou 
TABELA 2: Densidade Absoluta (DA), Frequência Relativa (FR), Altura Total e Diâmetro a Base do Caule (DBC) (média \pm desvios padrão) do componente de regeneração natural das espécies arbóreas de mangue nas áreas estudadas: Antonina (A1, A2 e A3) e Guaratuba (G1, G2 e G3), Paraná, Brasil.

TABLE 2: Absolute density (DA), Relative frequency (FA), Heigth, Diameter at steam base (DBC) (mean \pm standard deviation) for the regeneration component of the studied areas: Antonina (A1, A2 and A3) and Guaratuba (G1, G2 and G3), Paraná, Brasil.

\begin{tabular}{|c|c|c|c|c|c|c|c|c|}
\hline & A1 & $\mathrm{A} 2$ & A3 & média & G1 & G2 & G3 & média \\
\hline \multicolumn{9}{|c|}{ Densidade Absoluta } \\
\hline Avicennia schaueriana & 0 & 0 & 0,06 & 0,02 & 9,6 & 0,6 & 2,06 & 4,09 \\
\hline Laguncularia racemosa & 9 & 20,54 & 27,6 & 19,05 & 0,2 & 2,06 & 10,93 & 4,40 \\
\hline Rhizophora mangle & 4,14 & 0,86 & 2,20 & 2,4 & 0,67 & 7,27 & 1,2 & 3,05 \\
\hline Subtotal & 13,14 & 21,40 & 29,86 & 21,47 & 10,47 & 9,93 & 14,19 & 11,54 \\
\hline \multicolumn{9}{|c|}{ Frequência relativa } \\
\hline Avicennia schaueriana & 0 & 0 & 4,55 & 1,52 & 62,5 & 16,67 & 30,3 & 36,49 \\
\hline Laguncularia racemosa & 38,1 & 68,42 & 68,18 & 58,23 & 8,33 & 40 & 39,39 & 29,24 \\
\hline Rhizophora mangle & 61,9 & 31,58 & 27,27 & 40,25 & 29,17 & 43,33 & 30,3 & 34,27 \\
\hline Subtotal & 100 & 100 & 100 & & 100 & 100 & 100 & \\
\hline \multicolumn{9}{|c|}{ Altura } \\
\hline Avicennia schaueriana & - & - & 0,2 & 0,20 & $\begin{array}{c}0,22 \\
\pm 0,08\end{array}$ & $\begin{array}{c}0,16 \\
\pm 0,04\end{array}$ & $\begin{array}{c}0,55 \\
\pm 0,19\end{array}$ & 0,31 \\
\hline Laguncularia racemosa & $\begin{array}{c}0,1 \\
\pm 0,12\end{array}$ & $\begin{array}{c}0,1 \\
\pm 0,01\end{array}$ & $\begin{array}{c}0,1 \\
\pm 0,01\end{array}$ & 0,1 & $\begin{array}{c}0,08 \\
\pm 0,03\end{array}$ & $\begin{array}{c}0,1 \\
\pm 0,05\end{array}$ & $\begin{array}{c}0,12 \\
\pm 0,06\end{array}$ & 0,1 \\
\hline Rhizophora mangle & $\begin{array}{l}0,51 \\
\pm 0,2\end{array}$ & $\begin{array}{c}0,57 \\
\pm 0,22\end{array}$ & $\begin{array}{c}0,41 \\
\pm 0,21\end{array}$ & 0,5 & $\begin{array}{c}0,52 \\
\pm 0,11\end{array}$ & $\begin{array}{c}0,47 \\
\pm 0,13\end{array}$ & $\begin{array}{c}0,54 \\
\pm 0,19\end{array}$ & 0,51 \\
\hline \multicolumn{9}{|c|}{ Diâmetro da Base do Caule } \\
\hline Avicennia schaueriana & - & - & 0,3 & 0,3 & $\begin{array}{c}0,46 \\
\pm 0,14\end{array}$ & $\begin{array}{c}0,38 \\
\pm 0,07\end{array}$ & $\begin{array}{c}0,82 \\
\pm 0,27\end{array}$ & 0,55 \\
\hline Laguncularia racemosa & $\begin{array}{c}0,24 \\
\pm 0,25\end{array}$ & $\begin{array}{l}0,2 \\
\pm 0\end{array}$ & $\begin{array}{l}0,2 \\
\pm 0\end{array}$ & 0,21 & $\begin{array}{c}0,42 \\
\pm 0,04\end{array}$ & $\begin{array}{c}0,21 \\
\pm 0,05\end{array}$ & $\begin{array}{l}0,22 \\
\pm 0,1\end{array}$ & 0,28 \\
\hline Rhizophora mangle & $\begin{array}{c}1,43 \\
\pm 0,36\end{array}$ & $\begin{array}{c}1,4 \\
\pm 0,31\end{array}$ & $\begin{array}{c}1,23 \\
\pm 0,22\end{array}$ & 1,35 & $\begin{array}{l}1,41 \\
\pm 0,2\end{array}$ & $\begin{array}{c}1,24 \\
\pm 0,15\end{array}$ & $\begin{array}{c}1,38 \\
\pm 0,26\end{array}$ & 1,34 \\
\hline
\end{tabular}

Em que: $\mathrm{DA}=$ Densidade Absoluta $\left(\mathrm{n}^{0}\right.$ indivíduos. $\left.\mathrm{m}^{-2}\right) ; \mathrm{FR}=$ Frequência Relativa (\%), Altura $=$ altura total $(\mathrm{m})$; $\mathrm{DBC}=$ Diâmetro a Base do Caule $(\mathrm{cm})$.

que em Guaratuba, Avicennia schaueriana obteve a maior altura média, seguida por Rhizophora mangle e Laguncularia racemosa. Em Antonina, ocorreram os indivíduos mais altos de Rhizophora mangle, seguida por A. schaueriana e Laguncularia racemosa. Em ambos os manguezais, observouse uma grande variação no DAP dos indivíduos, refletindo altos valores dos desvios padrão para todas as espécies estudadas (Tabela 4). A PCoA sumarizou $75,8 \%$ da variância dos atributos fitossociológicos de plântulas, diferenciando mais fracamente os manguezais (Figura 2A). O eixo 1 sumarizou 46,9\% da variação dos dados, sendo os principais atributos responsáveis por essa variação o DBC e altura de Rhizophora mangle e altura e densidade absoluta de Laguncularia racemosa (Figura 2A, Tabela 5).

Em Antonina, a espécie com maior número de indivíduos nas classes de menor DAP foi Laguncularia racemosa, seguida de Rhizophora mangle. Em Guaratuba, a ocorrência de seis 
indivíduos com diâmetro acima de $30 \mathrm{~cm}$ de DAP foi marcante. Considerando-se todas as espécies, o manguezal de Antonina possui os maiores valores médios de altura e DAP que o manguezal de Guaratuba.

O número de indivíduos foi consideravelmente maior nas primeiras classes de diâmetro (1 a $15 \mathrm{~cm}$ ), principalmente em A1. O menor DAP foi registrado em Rhizophora mangle, com uma grande amplitude de valores, variando de $11,39 \mathrm{~cm}$, em Antonina a 2,41 cm, em Guaratuba. $\mathrm{O}$ maior número de indivíduos com DAP até $6 \mathrm{~cm}$ foi encontrado nas subáreas A1 e G1. Ainda em A1, ocorreu o maior número de indivíduos na classe 18 a $21 \mathrm{~cm}$. Os maiores valores de DAP foram observados em indivíduos vivos de Avicennia schaueriana, nas três subáreas de Antonina e nas subáreas G2 e G3, e em Laguncularia racemosa em G1.

Árvores mortas em pé foram encontradas em ambas as áreas, em todas as subáreas, com maior número em Guaratuba. O número de árvores mortas em pé variou consideravelmente entre as subáreas, sendo que G1 apresentou 5,6 vezes mais árvores mortas em pé do que A1 (Tabela 3).

Rhizophora mangle foi a espécie que se destacou com maior densidade, dominância, VC e VI em A1 e A2 (Tabela 3), enquanto que em A3, houve predomínio de indivíduos de Laguncularia racemosa, devido aos maiores valores de densidade, dominância, VC e VI. Em Guaratuba, A. schaueriana destacou-se com maiores índices em G1 e Rhizophora mangle em G2 (Tabela 3).
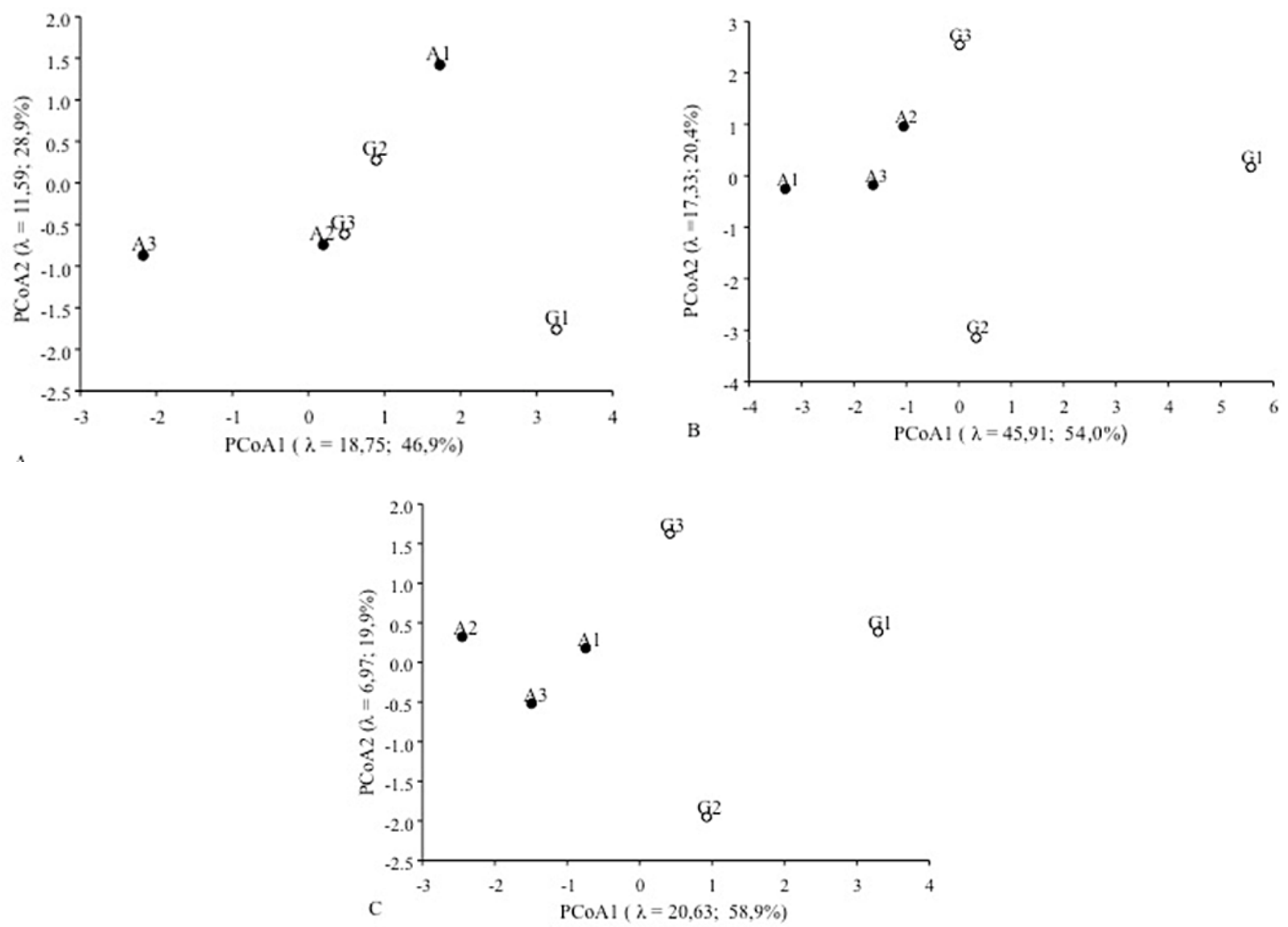

FIGURA 2: ACoP dos parâmetros fitossociológicos da regeneração natural (A), do componente arbóreo (B) e dos atributos pedológicos (C) dos manguezais de Antonina (A1, A2 e A3) e Guaratuba (G1, G2 e G3). Os autovalores e a respectiva porcentagem estão mostrados no gráfico. Somente as duas primeiras coordenadas são interpretáveis pelo critério de Broken Stick.

FIGURE 3: PCoA of phytossociological parameters of natural regeneration (A), tree component (B) and pedological attributes (C) of the mangroves from Antonina (A1, A2 and A3) and Guaratuba (G1, G2 and G3). The eigenvalues and the respective percentage are shown in the graphic. Only the first two coordinates are interpretable by the "Broken Stick" criterion. 
TABELA 3: Estrutura do componente arbóreo por espécie e por subárea, nas áreas de Antonina (A1, A2 e A3) e Guaratuba (G1, G2 e G3), Paraná, Brasil. DA: Densidade absoluta ( $n^{\circ}$ ind.ha-1), DoA: Dominância absoluta (g.ha $\left.{ }^{-1}\right)$, FA: Frequência absoluta (\%),VI: Valor de importância.

TABLE 3: Structural parameters for the tree component, by species and by subareas, from the studied areas: Antonina (A1, A2 and A3) and Guaratuba (G1, G2 and G3), Paraná, Brasil. DA: Absolute density $\left(\mathrm{n}^{\mathrm{o}}\right.$ ind.ha $\left.\mathrm{f}^{-1}\right)$, DoA: Dominance of the basal area $\left(\mathrm{g}^{\mathrm{h}} \mathrm{h}^{-1}\right)$, FA: absolute frequency (\%),VI: Importance value.

\begin{tabular}{|c|c|c|c|c|c|c|c|c|}
\hline & A1 & A2 & A3 & média & G1 & G2 & G3 & média \\
\hline \multicolumn{9}{|c|}{ Densidade Absoluta } \\
\hline Avicennia schaueriana & 100 & 160 & 400 & 220 & 2600 & 360 & 320 & 1093 \\
\hline Laguncularia racemosa & 2260 & 1160 & 1560 & 1660 & 620 & 880 & 1160 & 887 \\
\hline Rhizophora mangle & 2380 & 1420 & 920 & 1573 & 120 & 1840 & 940 & 967 \\
\hline Mortas em pé & 60 & 140 & 80 & 93 & 340 & 180 & 140 & 220 \\
\hline \multicolumn{9}{|c|}{ Dominância Absoluta } \\
\hline Avicennia schaueriana & 5,4 & 17,6 & 14,6 & 12,5 & 46,4 & 9,2 & 59 & 20,5 \\
\hline Laguncularia racemosa & 15,2 & 8,2 & 14,8 & 12,7 & 4,8 & 8,4 & 8,8 & 7,3 \\
\hline Rhizophora mangle & 19,6 & 7 & 11,0 & 12,5 & 20,06 & 10,2 & 2 & 10,75 \\
\hline Mortas em pé & 0,3 & 1,48 & 1,06 & 0,95 & 2,6 & 1,12 & 0,68 & 1,47 \\
\hline \multicolumn{9}{|c|}{ Frequência Absoluta } \\
\hline Avicennia schaueriana & 1,00 & 0,80 & 1,00 & 0,93 & 0,80 & 0,60 & 1,00 & 0,80 \\
\hline Laguncularia racemosa & 1,00 & 1,00 & 1,00 & 1,00 & 1,00 & 1,00 & 1,00 & 1,00 \\
\hline Rhizophora mangle & 0,40 & 1,00 & 1,00 & 0,80 & 1,00 & 1,00 & 1,00 & 1,00 \\
\hline Mortas em pé & 0,80 & 1,00 & 1,00 & 0,93 & 0,40 & 1,00 & 0,40 & 0,60 \\
\hline \multicolumn{9}{|c|}{ Valor de importância } \\
\hline Avicennia schaueriana & 48,85 & 92,55 & 82,99 & 74,80 & 194,50 & 77,47 & 130,36 & 134,11 \\
\hline Laguncularia racemosa & 118,23 & 98,61 & 122,67 & 113,17 & 59,54 & 90,54 & 91,25 & 80,44 \\
\hline Rhizophora mangle & 131,67 & 103,98 & 91,64 & 109,09 & 36,72 & 126,47 & 72,92 & 78,70 \\
\hline Mortas em pé & - & - & - & & - & - & - & \\
\hline
\end{tabular}

Em G3, apesar de Laguncularia racemosa ter maior densidade, Avicennia schaueriana teve maior dominância, VC e VI, em decorrência do maior porte (maior área basal, maior DAP) dos indivíduos analisados.

A PCoA do componente arbóreo (Figura 2B) mostrou que os dois eixos (PCoA1 e PCoA2) explicaram $74,4 \%$ da variância total das características analisadas. PCoA1 foi representada principalmente pela densidade para Avicennia schaueriana, densidade e dominância para Laguncularia racemosa e Rhizophora mangle. A PCoA2 foi representada principalmente pela área basal de Laguncularia racemosa (Tabela 5). A análise de PCoA do solo (Figura 2C) mostrou que os dois eixos (PCoA1 e PCoA2) explicaram 78,8\% da variância total das características analisadas. PCoA1 foi representada principalmente pela salinidade. PCoA2 foi representada principalmente pela matéria orgânica (Tabela 5). Segundo essa ordenação, é clara a distinção entre os solos dos manguezais de Antonina e Guaratuba.

\section{DISCUSSÃO}

Três espécies arbóreas ocorreram nos 
TABELA 4: Valores médios ( \pm desvio padrão) de altura $(\mathrm{m})$ e diâmetro a altura do peito (DAP, $\mathrm{cm})$ do componente arbóreo, por espécie e por subárea, nos manguezais de Antonina (A1, A2 e A3) e Guaratuba (G1, G2 e G3), Paraná, Brasil.

TABLE 4: Mean values ( \pm standard deviation) of height (Altura, $m$ ) and diameter at breast height (DAP, $\mathrm{cm}$ ) for the tree component, by species and by subareas, from the studied areas: Antonina (A1, A2 and A3) and Guaratuba (G1, G2 and G3), Paraná, Brazil.

\begin{tabular}{|c|c|c|c|c|c|c|c|c|}
\hline Espécies & A1 & $\mathrm{A} 2$ & A3 & Média & G1 & G1 & G3 & Média \\
\hline \multicolumn{9}{|c|}{ Altura (m) } \\
\hline $\begin{array}{l}\text { Avicennia } \\
\text { schaueriana }\end{array}$ & $\begin{array}{c}6,04 \\
\pm 4,23\end{array}$ & $\begin{array}{l}13,11 \\
\pm 1,54\end{array}$ & $\begin{array}{l}10,30 \\
\pm 4,54\end{array}$ & 9,82 & $\begin{array}{c}6,83 \\
\pm 4,44\end{array}$ & $\begin{array}{c}9,55 \\
\pm 5,49\end{array}$ & $\begin{array}{l}13,20 \\
\pm 2,15\end{array}$ & 9,86 \\
\hline $\begin{array}{l}\text { Laguncularia } \\
\text { racemosa }\end{array}$ & $\begin{array}{c}5,17 \\
\pm 3,42\end{array}$ & $\begin{array}{c}6,55 \\
\pm 3,66\end{array}$ & $\begin{array}{c}7,54 \\
\pm 4,34\end{array}$ & 6,42 & $\begin{array}{l}10,91 \\
\pm 2,94\end{array}$ & $\begin{array}{c}7,87 \\
\pm 3,28\end{array}$ & $\begin{array}{c}6,78 \\
\pm 2,78\end{array}$ & 8,52 \\
\hline $\begin{array}{l}\text { Rhizophora } \\
\text { mangle }\end{array}$ & $\begin{array}{c}7,43 \\
\pm 4,23\end{array}$ & $\begin{array}{c}7,04 \\
\pm 4,65\end{array}$ & $\begin{array}{l}13,33 \\
\pm 1,73\end{array}$ & 9,27 & $\begin{array}{c}3,13 \\
\pm 4,48\end{array}$ & $\begin{array}{c}7,96 \\
\pm 4,15\end{array}$ & $\begin{array}{c}3,51 \\
\pm 3,63\end{array}$ & 4,87 \\
\hline Média & 6,21 & 8,90 & 10,39 & 8,50 & 6,96 & 8,46 & 7,83 & 7,75 \\
\hline \multicolumn{9}{|c|}{$\mathrm{DAP}(\mathrm{cm})$} \\
\hline $\begin{array}{l}\text { Avicennia } \\
\text { schaueriana }\end{array}$ & $\begin{array}{c}19,75 \\
\pm 19,21\end{array}$ & $\begin{array}{c}27,35 \\
\pm 23,97\end{array}$ & $\begin{array}{c}16,02 \\
\pm 14,92\end{array}$ & 21,04 & $\begin{array}{c}8,82 \\
\pm 12,19\end{array}$ & $\begin{array}{c}14,54 \\
\pm 11,42\end{array}$ & $\begin{array}{c}38,06 \\
\pm 37,32\end{array}$ & 20,47 \\
\hline $\begin{array}{l}\text { Laguncularia } \\
\text { racemosa }\end{array}$ & $\begin{array}{c}7,23 \\
\pm 5,79\end{array}$ & $\begin{array}{c}7,98 \\
\pm 5,99\end{array}$ & $\begin{array}{c}7,19 \\
\pm 4,96\end{array}$ & 7,47 & $\begin{array}{c}9,12 \\
\pm 4,04\end{array}$ & $\begin{array}{c}9,82 \\
\pm 4,64\end{array}$ & $\begin{array}{c}9,13 \\
\pm 5,01\end{array}$ & 9,36 \\
\hline $\begin{array}{l}\text { Rhizophora } \\
\text { mangle }\end{array}$ & $\begin{array}{c}8,21 \\
\pm 6,09\end{array}$ & $\begin{array}{c}6,28 \\
\pm 4,44\end{array}$ & $\begin{array}{l}11,39 \\
\pm 4,73\end{array}$ & 8,63 & $\begin{array}{c}2,41 \\
\pm 0,60\end{array}$ & $\begin{array}{c}7,29 \\
\pm 4,43\end{array}$ & $\begin{array}{c}4,56 \\
\pm 3,93\end{array}$ & 4,75 \\
\hline Média & 11,73 & 13,87 & 11,53 & 12,38 & 6,78 & 10,55 & 17,25 & 11,53 \\
\hline
\end{tabular}

manguezais estudados: Avicennia schaueriana, Laguncularia racemosa e Rhizophora mangle, de acordo com o esperado. Estudos semelhantes relataram a ocorrência destas espécies em toda a faixa de manguezais da costa brasileira, porém, a distribuição destas pode diferir e está relacionada com as condições ambientais e o estabelecimento dos propágulos (BERNINI e REZENDE, 2010; CUNHA-LIGNON, 2001; SCHAEFFERNOVELLI et al., 1990). Segundo a classificação de Schaeffer-Novelli et al. (1990), nos manguezais pertencentes à zona VII, não há ocorrência de outras espécies de mangue, devido às condições climáticas peculiares da região subtropical.

A alta variabilidade entre as parcelas do componente de regeneração natural dentro de cada área refletiu na análise de PCoA, que mostrou de maneira mais fraca as diferenças entre os manguezais estudados, quando comparada com a análise do componente arbóreo.

Enquanto as plântulas de Laguncularia racemosa são mais abundantes e bem desenvolvidas no manguezal de Antonina, indivíduos jovens de Rhizophora mangle se destacam por apresentarem maior porte em Guaratuba. A localização das áreas de estudo parece influenciar o estabelecimento de novos propágulos e, consequentemente, ocasiona diferenças na ocorrência das espécies no componente de regeneração. Antonina está dentro do estuário, com maior aporte de matéria orgânica e mais protegida em relação à Guaratuba, que se localiza mais próxima da desembocadura da Baía de Guaratuba, estando sujeita à ação das correntes de marés. O tamanho dos propágulos e a flutuabilidade são fatores determinantes para o estabelecimento e desenvolvimento dos manguezais (CUNHALIGNON, 2006). Em áreas mais internas da baía é mais difícil haver a dispersão de propágulos 
TABELA 5: Correlação entre os valores dos atributos fitossociológicos da regeneração natural, do componente arbóreo, do solo e dos escores das duas coordenadas principais entre os manguezais de Antonina e Guaratuba. DAP: Diâmetro altura do peito; DA: Densidade absoluta; DomA: Dominância absoluta; DBC: Diâmetro da base do caule; MO: Matéria orgânica; CTC: Capacidade de troca catiônica; Eh: Potencial redox.

TABLE 5: Correlation among values of phytossociological parameters of natural regeneration component and tree component, of soil and of scores of the two principal coordinates from the analysis between Antonina and Guaratuba, Paraná, Brasil. Altura: height; DAP: Diameter at breast height; Área Basal: Basal area; DA: Absolute density; DomA: Dominance of the basal area; DBC: Diameter at stem base; MO: Organic matter; CTC: Cation exchange capacity; Eh: Redox potential; Salinidade: Salinity.

\begin{tabular}{|c|c|c|c|}
\hline \multirow{2}{*}{\multicolumn{2}{|c|}{ Variáveis fitossociológicas do componente de regeneração }} & \multicolumn{2}{|c|}{ Coordenada Principal } \\
\hline & & 1 & 2 \\
\hline \multirow{3}{*}{ Laguncularia racemosa } & Altura & $-0,901$ & 0,153 \\
\hline & $\mathrm{DBC}$ & 0,490 & 0,570 \\
\hline & DA & $-0,823$ & 0,347 \\
\hline \multirow{3}{*}{ Rhizophora mangle } & Altura & 0,675 & 0,016 \\
\hline & DBC & 0,862 & 0,420 \\
\hline & DA & $-0,190$ & 0,778 \\
\hline \multirow{2}{*}{\multicolumn{2}{|c|}{ Variáveis fitossociológicas do componente arbóreo }} & \multicolumn{2}{|c|}{ Coordenada Principal } \\
\hline & & 1 & 2 \\
\hline \multirow{7}{*}{ Avicennia schaueriana } & Altura & $-0,148$ & 0.388 \\
\hline & DAP & $-0,601$ & 0.598 \\
\hline & Área basal & $-0,467$ & 0.713 \\
\hline & DA & 0,943 & -0.044 \\
\hline & DomA & 0,674 & 0.652 \\
\hline & Altura & 0,852 & -0.378 \\
\hline & DAP & 0,649 & -0.442 \\
\hline \multirow[t]{2}{*}{ Laguncularia racemosa } & Área basal & $-0,056$ & 0.829 \\
\hline & DA & $-0,925$ & 0.129 \\
\hline \multirow{6}{*}{ Rhizophora mangle } & DomA & $-0,917$ & -0.065 \\
\hline & Altura & $-0,844$ & -0.342 \\
\hline & DAP & $-0,844$ & -0.380 \\
\hline & Área basal & $-0,899$ & -0.257 \\
\hline & DA & $-0,909$ & -0.228 \\
\hline & DomA & $-0,947$ & -0.274 \\
\hline \multirow{2}{*}{\multicolumn{2}{|c|}{ Variáveis do solo }} & \multicolumn{2}{|c|}{ Coordenada Principal } \\
\hline & & 1 & 2 \\
\hline & MO & $-0,128$ & $-0,944$ \\
\hline & $\mathrm{CTC}$ & $-0,884$ & $-0,245$ \\
\hline & Salinidade & 0,956 & 0,077 \\
\hline & Eh & 0,296 & $-0,468$ \\
\hline
\end{tabular}

maiores, como de Rhizophora mangle, enquanto que em áreas mais permanentemente inundadas, o estabelecimento de propágulos maiores é favorecido (RABINOWITZ, 1978).
A grande ocorrência de plântulas de Laguncularia racemosa, em Antonina, também pode ser explicada pelo sucesso no estabelecimento, pois apresentam rápido enraizamento e baixa 
sensibilidade à ação da maré (DELGADO et al., 2001) e benefício no desenvolvimento pelo aporte extra de matéria orgânica (PERGENTINO et al., 2005). Além disso, estudos de regeneração em manguezais reportam maior número de plântulas em ambientes mais impactados, como no caso da área de Antonina, a qual possui histórico de intervenção antrópica como as atividades portuárias, derramamento de óleo, dragagem, esgoto doméstico, entre outros (NOERNBERG et al., 2008).

A baixa frequência de plântulas de Avicennia schaueriana em Antonina parece ser influenciada pelo número reduzido de indivíduos adultos encontrados, ou ainda, ao padrão de dispersão da espécie. Em geral, a distribuição das plântulas de Avicennia schaueriana ocorre ao redor de árvores adultas e em depressões protegidas do movimento de água, limitando o seu estabelecimento em regiões com menor influência da maré (DELGADO et al., 2001).

Tanto para plântulas quanto para indivíduos adultos, as espécies do gênero Avicennia possuem mecanismos para a sobrevivência em locais com substrato altamente salino ou ambientes de baixas temperaturas e são mais tolerantes ao estresse ambiental (PETRI, 2011). Em contrapartida, essa espécie é capaz de atingir melhor desenvolvimento em locais com menor alteração ambiental de origem antrópica (CINTRÓN-MOLERO e SCHAEFFERNOVELLI, 1992). Esse é o caso de Guaratuba, onde ocorrem indivíduos de Avicennia schaueriana com maior área basal e maior diâmetro. Outros estudos semelhantes indicaram que essa espécie tem grande tolerância à salinidade, desenvolvendo-se melhor em ambientes com maior salinidade (SERGIO et al., 2006). Em ambientes de hipersalinidade, no entanto, as espécies têm seu desenvolvimento prejudicado e apresentam menor porte (TWILLEY e CHEN, 1998). $\mathrm{Na}$ área de Guaratuba, onde a espécie é dominante, além da maior salinidade na água intersticial, o solo das subáreas G1 e G3 apresentou material mineral e horizonte glei dentro dos primeiros $50 \mathrm{~cm}$, onde, em geral, possui elevados teores de sais e de sódio (EMBRAPA, 2009).

No componente arbóreo, as diferenças na estrutura entre as áreas parecem estar relacionadas com as diferentes respostas adaptativas que as espécies apresentam ao ambiente em que estão inseridas. A salinidade, uma das principais características do solo responsável pela distinção das áreas (Tabelas 1 e 5), afeta o desempenho das plantas pelo deficit de água, toxidez por íons que provocam desequilíbrio nutricional e indiretamente atua mediando competições interespecíficas (ESTEVES e SUZUKI, 2008). Estudos nos mangues do Espírito Santo registraram árvores de menor porte quando os teores de salinidade eram mais elevados (SILVA et al., 2005).

Os indivíduos das espécies estudadas apresentaram altura e DAP similares aos encontrados por outros estudos da região Sul e sudeste do Brasil (SESSEGOLO, 1997; PEREIRA et al., 2009). Entretanto, esses valores são inferiores aos encontrados em regiões tropicais (SCHAEFFERNOVELLI et al., 1995). A posição geográfica dos manguezais influencia na estruturação dos bosques de mangue (SCHAEFFER-NOVELLI et al., 1990). Ao Norte da costa brasileira, os manguezais são menos escassos e de maior porte, em relação ao sul do país. A diferença entre as populações situadas mais ao norte do Brasil pode ser reflexo de mudanças climáticas ocorridas no Quaternário, período em que eram frequentes as oscilações de temperatura (PIL et al., 2011). Ainda, regiões mais próximas da linha do Equador sofrem maior amplitude de marés e temperaturas (SCHAEFFER-NOVELLI et al., 1990), possibilitando o desenvolvimento de indivíduos de maior porte.

Pela análise de PCoA, a densidade absoluta das espécies do componente arbóreo é um dos parâmetros que mais influencia na separação das áreas, reforçando a relação entre salinidade do solo e a ocorrência das espécies (Tabela 5). Avicennia shaueriana, relatada como a espécie mais tolerante à salinidade, tem seu valor de densidade absoluta mais correlacionado com a separação das áreas, assim como a salinidade na análise de solo (Tabela 5). Smith III (1992) sugere que a baixa densidade de troncos, juntamente com as maiores alturas e alta taxa de área basal viva, caracterizam bosques mais estruturalmente complexos. Apesar de se observar a baixa densidade de indivíduos, quando comparada com estudos semelhantes (PEREIRA et al., 2009), não é possível afirmar que os bosques de mangue apresentam grande desenvolvimento em termos de altura. O principal indício é a ocorrência de manchas estruturalmente bem desenvolvidas, com alturas médias e DAP superiores ao de outros indivíduos, das três espécies, dentro das subáreas, formando um mosaico. Este fato pode explicar a diferença entre as áreas, uma vez que o tamanho dessas manchas diferem dentro das subáreas e em ambas as áreas.

Assim como outros autores relataram 
(BERNINI e REZENDE, 2004; 2010; SILVA et al., 2005), estudos fitossociológicos em áreas de manguezais são importantes para estabelecer estratégias de manejo e conservação deste ecossistema. De acordo com os resultados encontrados, áreas geograficamente próximas apresentaram uma variabilidade na vegetação em decorrência das diferentes características ambientais. A salinidade e a matéria orgânica são os atributos do solo que diferenciaram os manguezais de Antonina e Guaratuba em dois grupos, influenciando no perfil estrutural da vegetação, de forma que a densidade e altura das espécies diferiram entre as áreas estudadas. Os resultados refletem as particularidades dos manguezais estudados, ressaltando a necessidade de ações específicas de manejo e conservação deste ecossistema.

\section{CONCLUSÃO}

O manguezal de Antonina diferiu estruturalmente do manguezal de Guaratuba, tanto na regeneração natural quanto no componente arbóreo. As diferenças estruturais da regeneração natural entre as áreas são atribuídas ao diâmetro e altura de Rhizophora mangle e à altura e densidade absoluta de Laguncularia racemosa. A variação do componente arbóreo entre as áreas refere-se à densidade de Avicennia schaueriana e a densidade e dominância de Laguncularia racemosa e Rhizophora mangle. Entre as características edáficas estudadas dos manguezais, a salinidade e a matéria orgânica foram as mais importantes na separação entre as áreas.

\section{AGRADECIMENTOS}

À Petrobras e Fundação Araucária (Convênio 412/09 protocolo 12499) pelo apoio financeiro. À Coordenação de Aperfeiçoamento de Pessoal de Nível Superior (CAPES) pela concessão de bolsas e ao Conselho Nacional de Desenvolvimento Científico e Tecnológico (CNPq) pela bolsa de produtividade (301561/2010-9) concedida à segunda autora.

\section{REFERÊNCIAS BIBLIOGRÁFICAS}

BERNINI, E.; REZENDE, C.E. Estrutura da vegetalção em florestas de mangue do estuário do rio Paraíba do Sul, Estado do Rio de Janeiro, Brasil. Acta bot. Bras., Feira de Santana, v.18, n.3, p.491-502, 2004.

BERNINI, E.; REZENDE, C.E. Variação estrutural em florestas de mangue do estuário do rio Itabapoana, ES-RJ. Biotemas, Florianópolis, v.23, n.1, p.49-60, 2010.

BIGARELLA, J.J. Contribuição ao estudo da planície litorânea do Estado do Paraná. Arquivos Brasileiros de Biologia e Tecnologia, Curitiba, v.1, p.75-111, 1946.

BOEGER, M. R. T. et al. Diagnóstico da vegetação de manguezal. In: Ostrensky, A. (Org.). Diagnóstico ictiofaunístico em ambientes afetados por vazamento de óleo na Serra do Mar, no estado do Paraná. Curitiba: FUNPAR, 2011. p. 1-184.

CITRÓN, G.; SCHAEFFER-NOVELLI, Y. Introducción a la ecologia del manglar. San Juan:Rostlac, 1983. 109p.

CINTRÓN-MOLERO, G.; SCHAEFFERNOVELLI, Y. Ecology and management New World mangroves.. In: SEELIGER, U. (Ed.). Coastal Plant Communities of Latin América. San Diego: Academic Press. 1992. p. 233-258

CUNHA-LIGNON, M. Dinâmica do manguezal no Sistema de Cananéia- Iguape, Estado de São Paulo - Brasil. 2001. 105p. Dissertação (Mestrado em Ciências) - Universidade de São Paulo, São Paulo, 2001.

CUNHA-LIGNON, M. Variação espaço-temporal de bosques de mangue III Simpósio Regional de Geoprocessamento e Sensoriamento Remoto. 2006. $5 \mathrm{p}$.

DELGADO, P. et al. The importance of propagule establishment and physical factors in mangrove distributional patterns in a Costa Rican estuary. Aquatic Botany, Amsterdam, v.71, p.157-178, 2001.

EMBRAPA - Empresa Brasileira de Pesquisa Agropecuária. Sistema Brasileiro de Classificação de Solos. Rio de Janeiro : EMBRAPA-SPI. 2009. 412p.

ESTEVES, B. dos S.; SUZUKI, M. S. Efeito da salinidade sobre as plantas. Oecol. Bras., Rio de Janeiro, v.12,n.4, p. 662-679, 2008.

GOWER, J. C. Some distance properties of latent root and vector methods used in multivariate analysis. Biometrika, Oxford, v.53, p.325-338, 1966.

IBGE - Instituto Brasileiro de Geografia e Estatística. Manual técnico da vegetação brasileira. Série Manuais Técnicos em Geociências. Rio de Janeiro: IBGE. 1992. p. 9-38.

LANA, P. C. Manguezais: diagnóstico, conflitos 
e prognósticos. In: LIMA, R.E; NEGRELLE, R. (Eds.). Meio Ambiente e desenvolvimento no litoral do Paraná. Diagnóstico. Curitiba: Editora da Universidade Federal do Paraná. 1998. p.105-118.

KATHIRESAN, K.; BINGHAM, B.L. Biology of Mangroves and Mangrove Ecosystems. Advances in Marine Biology, Plymouth, v.40, p.81-251, 2001.

MAACK, R. 1981. Geologia e Geografia da região de Vila Velha, Estado do Paraná e considerações sobre a glaciação carbonífera no Brasil. Arquivos do Museu Paranaense, Curitiba, v.5, p.1-46, 1946. MUELLER-DOMBOIS, D.; ELLENBERG, H. Aims and methods of vegetation ecology. New York: Wiley. 1974. 574p.

NOERNBERG, M.A. et al. Determinação da sensibilidade do litoral paranaense à contaminação por óleo. Brazilian Journal of Aquatic Science and Technology, Itajaí, v.12, n.2, p.49-59, 2008.

OKSANEN, J. et al. 2008. Vegan: Community Ecology Package. R package version 1.15-1. http://cran.r-project.org/, http://vegan.r-forge.rproject.org/

PEREIRA, F.V. et al. Estrutura da vegetação em duas áreas com diferentes históricos de antropização no manguezal de Anchieta, ES. Boletim do Laboratório de Hidrobiologia, Maranhão, v.22, p.1-8, 2009.

PERGENTINO, T.C; LANDIM, M.F. Distribuição espacial de plântulas de espécies arbóreas de manguezais. In: VII Congresso de Ecologia do Brasil, Minas Gerais. 2005. Disponível em http://www.seb-ecologia.org.br/viiceb/listaresumo. html (acesso em 10/04/2010).

PETRI, D. J. C. et al. Distribuição das espécies e estrutura do manguezal do rio Benevente, Anchieta, ES. Biota Neotropica, Campina, v.11, n.3, p. 107116, 2011.

PIL, M. W. et al. Postglacial north-south expansion of populations of Rhizophora mangle (Rhizophoraceae) along the Brazilian coast revealed by microsatellite analysis. American Journal of Botany, St. Louis, v.98, n.6, p.1031-1039, 2011.

$\mathrm{R}$ Development Core Team. 2009. $R$ version 2.9.0.P Project for Statistical Computing, Vienna, Austria. (www.r-project.org)
RABINOWITZ, D. Early growth of mangrove seedlings in Panamá, and an hypothesis concerning the relationship of dispersal and zonation. Journal of Biogeography, Oxford, v. 5, p.113-133, 1978. ROBERTS, D.W. 2007. Labdsv: Ordination and Multivariate Analysis for Ecology. R package version 1.3-1, URL http://CRAN.R-project.org/ package $=$ labdsv.

SCHAEFFER-NOVELLI, Y. et al. Variability of the mangroves ecosystem along the Brasilian coast. Estuaries, Port Republic, v.13, n.2, p.201-218, 1990.

SCHAEFFER-NOVELLI, Y. Manguezais brasileiros. 1991. 42p. Tese de Livre Docência. Instituto Oceanográfico da Universidade de São Paulo, São Paulo, 1991.

SCHAEFFER-NOVELLI, Y. Manguezal: ecossistema entre a terra e o mar. São Paulo, Caribbean Ecological Research. 1995. 64p.

SCHAEFFER-NOVELLI, Y. Grupo de ecossistemas: manguezal, marisma e apicum. São Paulo: Caribbean Ecogical Research. 2000. 119p.

SÉRGIO, C. et al. Caracterização morfofisiológica do manguezal de Cananéia - SP. In: Environmental and Health World Congress, Anais. Santos: 2006.

SESSEGOLO, G.C.S. Estrutura e produção de serapilheira do manguezal do Rio Baguaçu, Baía de Paranaguá-PR. 1997. 130p. Dissertação (Mestrado em Oceanografia) - Universidade Federal do Paraná, Curitiba. 1997.

SILVA, M.A.B.; BERNINI, E.; CARMO, T.M.S. Características estruturais de bosques de mangue do estuário do rio São Mateus, ES, Brasil. Acta Botanica Brasilica, Feira de Santana, v.19, n.3, p.465-471, 2005.

SMITH III, T. J., Forest Structure. In: ROBERTSON, A. I.; ALONGI, D. M. (Eds.). Coastal and Estuarine Studies. Washington D.C.: American Geophysical Union, 1992. p.101-136.

STATSOFT Inc., 2005. STATISTICA (data analysis software system) version 7.1 for Windows: statistics. STATSOFT, Inc., Tulsa, USA.

TWILLEY, R.R., CHEN, R. A water budget and hydrology model of a basin mangrove forest in Rookery Bay, Florida. Marine and Freshwater Research, Melbourne, v.49, p.309-323, 1998. 